EPJ Web of Conferences 60, 16010 (2013)

DOI: $10.1051 /$ epjconf $/ 20136016010$

(C) Owned by the authors, published by EDP Sciences, 2013

\title{
Single top quark production with CMS
}

\author{
Davide Piccolo ${ }^{1, a}$ for the CMS Collaboration \\ ${ }^{1}$ Laboratori Nazionali di Frascati dell'INFN, Italy
}

\begin{abstract}
Measurements of single top quark production performed using the CMS experiment [1] data collected in 2011 at centre-of-mass energies of $7 \mathrm{TeV}$ and in 2012 at $8 \mathrm{TeV}$, are presented. The cross sections for the electroweak production of single top quarks in the t-channel and in association with W-bosons is measured and the results are used to place constraints on the CKM matrix element $\mathrm{V}_{t b}$. Measurements of top quark properties in single top quark production are also presented. The results include the measurement of the charge ratio in the single top t-channel.
\end{abstract}

\section{Introduction}

The top quark is by far the heaviest quark and the heaviest particle ever observed. This high mass close to the Electroweak scale makes the top quark studies extremely interesting. Moreover the top quark decays faster than the hadronization time scale so this represents an unique opportunity to explore the "bare" quark properties.

Top quark can be produced at an hadron collider either in couple $t \bar{t}$ and as single top via Electroweak processes. Three main processes are responsible of single top quark production: $\mathrm{t}$-channel, associated production of $\mathrm{t}$ and $\mathrm{W}$ boson, s-channel. Figure. 1 shows the leading order diagrams of the three processes.

At LHC the expected cross section for the three processes are the following[2]:

$$
\begin{gathered}
\sigma_{t-c h .}=64.57 p b(87.8 p b) \\
\sigma_{t W}=15.6 p b(22.4 p b) \\
\sigma_{s-c h .}=4.59 p b(5.6 p b) .
\end{gathered}
$$

at $\sqrt{s}=7 \mathrm{TeV}(8 \mathrm{TeV})$.

In order to discriminate the signal from the background only the leptonic decays of the $\mathrm{W}$ boson are analyzed, so in all the analysis one or two isolated leptons and missing transverse energy are required in the events. In addition according to the different channels one or two reconstructed jets are required and one has to be identified as coming from $b$ quarks fragmentation according to specific b-tagging algorithms[3].

\footnotetext{
ae-mail: dpiccolo@lnf.infn.it
}

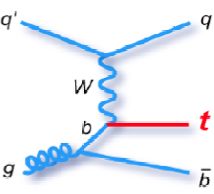

t-channel

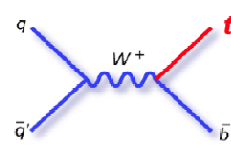

s-channel

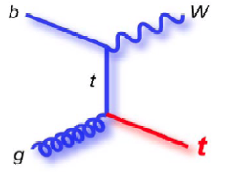

tW-channel
Figure 1. Leading order diagrams for the single top production

\section{2 t-channel cross section}

CMS has measured the t-channel cross section for a LHC center of mass energy of both 7 and $8 \mathrm{TeV}$ [4][5].

The most important background processes whose signatures fake the t-channel signal are $\mathrm{W}+\mathrm{Jets}$ in which one of the jets is a b-jet, $t \bar{t}$ events in which one top decays hadronically and the other one semi-leptonically and with lower statistics Z+jets, VV, QCD and other single top channels.

The signature of the t-channel is characterized by the presence of a single isolated lepton, one central b-jet coming from the top quark decay, an additional light jet coming from the recoil of the proton (usually emitted at high psedudorapidity) and momentum imbalance due to the presence of the neutrino. In order to discriminate the signal from these backgrounds a dedictaed selection is optimized to enrich the sample with t-channel events:

- lepton selection: exactly one muon with transverse momentum greater than $20 \mathrm{GeV} / \mathrm{c}(26 \mathrm{GeV} / \mathrm{c}$ at $8 \mathrm{TeV})$ and $|\eta|<2.1$ or one electron with transeverse momentum greater than $30 \mathrm{GeV} / \mathrm{c}$ and $|\eta|<2.5$ is required to pass tight quality and isolation criteria. After this step the QCD bakcground is almost rejected;

- jet selection: exactly two jets are required to have $p_{t}>$ $30 \mathrm{GeV} / \mathrm{c}$ (at $8 \mathrm{TeV}$ this cut is increased to $60 \mathrm{GeV} / \mathrm{c}$ to reduce pile-up events) for $|\eta|<4.5$. 
- b-tagging: one and only one jet is required to come from b-quark fragmentation, that is it should pass a tight threshold on the Track Counting High-Purity (TCHP) b-tagging algorithm [3]. After this requirement $\mathrm{W}+$ jets and $t \bar{t}$ backgrounds whose final states is characterized by the presence of two b-jets, are heavily reduced.

- W and top mass constraints: The $\mathrm{W}$ transverse mass $m_{T}$ is required to be $>40 \mathrm{GeV} / \mathrm{c}^{2}$ for $7 \mathrm{TeV}$ center-ofmass energy or $50 \mathrm{GeV} / \mathrm{c}^{2}$ for $8 \mathrm{TeV}$ (muon channel), or $E_{T}^{\prime}>35 \mathrm{GeV}$ (electron channel). Finally a cut is applied on the reconstructed top-quark mass defining a Signal Region (SR) for $130<m_{l v b}<220 \mathrm{GeV} / \mathrm{c}^{2}$ and a $\mathrm{W}+$ jets and tt enriched Sideband Region (SB) outside the mass window.

The cross section has been measured with and integrated luminosity of $1.17 \mathrm{fb}^{-1}\left(1.56 \mathrm{fb}^{-1}\right)$ in the muon (electron) channel for the $7 \mathrm{TeV}$ center of mass energy and with $5.0 \mathrm{fb}^{-1}$ in the muon channel for the $8 \mathrm{TeV}$ center of mass. In order to extract the cross section, three independent approaches are used: $\left|\eta_{j^{\prime}}\right|$ analysis, robust and with little model dependence, and two multi-variate analysis (Neural network and Boosted Decision Tree) for the precise measurement.

\section{$2.1\left|\eta_{j^{\prime}}\right|$ analysis}

The $\left|\eta_{j^{\prime}}\right|$ analysis explore the characteristic distribution of the pseudorapidity of the recoiling quark that is emitted usually at high $\eta$ values. Several categories are defined according to the number of jets above a given $\mathrm{p}_{t}$ and the number of jets identified as coming from $b$-quark. The signal region is the region with 2 Jets with $\mathrm{p}_{t}>30 \mathrm{GeV} / \mathrm{c}$ and exacty one of them tagged as b-jet. Categories 2 Jets 0 tags and $2 / 3$ Jets and 2 tags are enriched of $\mathrm{W}+$ jets and $t \bar{t}$ events respectively. The signal is extracted performing a Maximum likelihood fit to the pseudorapidity of the forward jet, $\left|\eta_{j^{\prime}}\right|$ template distribution, in the signal region. Background yield are extracted by the fit as well, after having modeled them with 1-dimensional histograms either from simulation or from data-driven estimations. Fig. 2 shows the distribution of the $\left|\eta_{j^{\prime}}\right|$ variable defined as the pseudorapidity of the recoiling jet for the $\mathrm{MC}$ and with the results of the fit on data.

\subsection{Multivariate analysis}

A multivariate approach permits the precise measurement of the t-channel cross section by optimizing the signal selection vs the backgrounds. Two independent analysis are performed. A first approach uses 37 (38) variables in the muon (electron) channel to build a Neural Network (NN) output to discriminate the signal from the background in 6 independent regions corresponding to $2 / 3 / 4$ jets and $1 / 2$ b-tagged jets. The Neural network output is shown in fig. 3 for the muon channel in signal region ( 2 jets, 1 tag) comparing data and MC. A clear enhancement of the signal over the background is visible for values around one. A second approach uses 11 variables to construct a Boosted Decision Tree (BDT) output separately for the

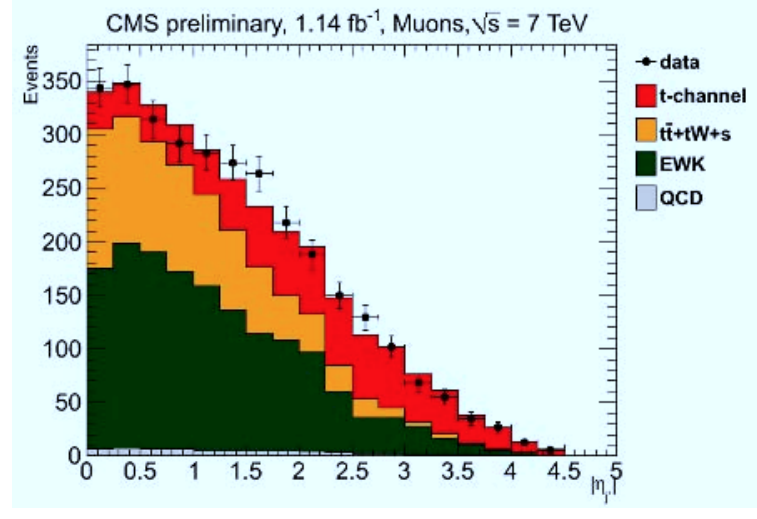

Figure 2. $\left|\eta_{j^{\prime}}\right|$ distribution in the muon channel in the signal region optimized for the t-channel analysis

electron and muon channels in the signal enriched (2-jets 1-tag) and background enriched (3-jets 1-tag) categories. The NN and BDT analyses employ a Bayesian approach [6] to measure the single-top- quark production cross section. The signal cross section is extracted simultaneously from the data distributions of the corresponding multivariate discriminator, modelled as 1-dimensional histograms, in the different categories. The signal yield is parameterized in terms of the signal strength which is defined as the actual cross section divided by the SM prediction.

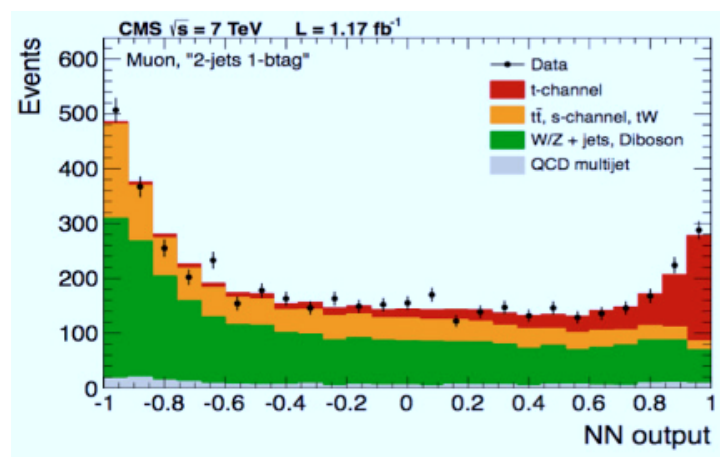

Figure 3. Neural Network output distribution in the muon channel in the signal region optimized for the t-channel analysis

\section{3 t-channel cross-section combination at $7 \mathrm{TeV}$}

The three analysis are combined with the BLUE method [7]. The statistical correlation between the analysis is between $60 \%$ and $75 \%$. The correlation assumed for jet/ $\mathrm{E}_{t}^{\text {miss }}$ related systematic uncertainty between MVA analysis and $\left|\eta_{j^{\prime}}\right|$ analysis, is $20 \%$. All the other systematic are assumed to be $100 \%$ correlated.

The combined result of the measured cross section at center of mass energy of $7 \mathrm{TeV}$ is [4]: 


$$
\begin{array}{r}
\left.\left.\sigma_{t-c h}=67.2 \pm 3.7 \text { (stat. }\right) \pm 3.0 \text { (syst. }\right) \\
\pm 3.5 \text { (theor. }) \pm 1.5 \text { (lum.) pb }
\end{array}
$$

In the assumption that $\left|\mathrm{V}_{t b}\right|$ is much larger than $\left|\mathrm{V}_{t s}\right|$ and $\left|\mathrm{V}_{t d}\right|$ and assuming the standard Model it is possible to extract $\left|\mathrm{V}_{t b}\right|$ from the following formula:

$$
\left|V_{t b}\right|=\sqrt{\sigma_{t-c h} / \sigma_{t-c h}^{\text {theo. }}}=1.02 \pm 0.05 \pm 0.02 \text { (theo.) }
$$

which represent a measurement at $5 \%$ level of $\left|\mathrm{V}_{t b}\right|$. or

$$
\left|V_{t b}\right|>0.92 @ 95 \% C L \text { for }\left|V_{t b}\right| \text { in }[0,1]
$$

The systematic uncertainties having the most relevant impact on the measurement are the modeling of $\mathrm{W}+$ jets background and the difference between powheg and compHEP in the MC signal generator.

\section{4 t-channel cross section at $8 \mathrm{TeV}$}

The measurement of the t-channel cross section has been repeated at $8 \mathrm{TeV}$ on a data sample of $5 \mathrm{fb}^{-1}$ in the muon channel only [5]. The selection criteria are very similar to those used at $7 \mathrm{TeV}$ with some additional requirement to reduce the effect of the increased pile-up. In this analysis only the $\left|\eta_{j^{\prime}}\right|$ analysis has been used for a cross section result of

$$
\sigma_{t-c h}=80.1 \pm 5.7(\text { stat. }) \pm 11.0(\text { syst. }) \pm 4(\text { lum. }) p b
$$

and

$$
\left|V_{t b}\right|=0.96 \pm 0.08 \pm 0.02 \text { (theo.) }
$$

or

$$
\left|V_{t b}\right|>0.81 @ 95 \% C L \text { for }\left|V_{t b}\right| \text { in }[0,1]
$$

The Jet Energy scale is the main contribution to the systematic error together with the MC signal generator.

In figure 4 the experimental results at 7 and $8 \mathrm{TeV}$ are compared with the Standard Model expectations.

\section{3 tW-channel cross section}

One of the leading order Feynman diagram for the associated production of single top quark with a $\mathrm{W}$ boson is shown in fig. $1 \mathrm{c}$ ). At the NLO the definition of the process mixes with the $t \bar{t}$ channel. Two approaches are usually proposed to define the $\mathrm{tW}$ signal: diagram removal schema (DR) [8] and diagram subtraction schema [9]. In this analysis the first schema has been adopted and the difference with respect to the second one is included as a systematic effect. The analysis [10] is performed on a data sample of $4.9 \mathrm{fb}^{-1}$ at $7 \mathrm{TeV}$ center of mass energy. To select signal events, two leptons (muons or electrons) with opposite

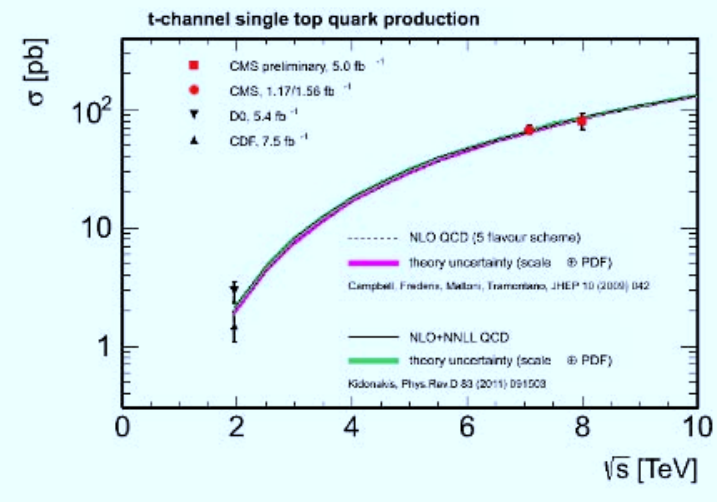

Figure 4. Single top t-channel cross section as function of the centre-of-mass energy. The error band (width of the curve) is obtained by varying the top mass within its current uncertainty [11], estimating the PDF uncertainty according to the HEPDATA recommendations [12], and varying the factorization and renormalization scales coherently by a factor two up and down.

charge are required and exactly one jet with $\mathrm{p}_{t}>30 \mathrm{GeV} / \mathrm{c}$ and tagged as a b-jet. Additional cuts on the invariant mass of the two leptons are applied in order to reduce the background coming from di-leptons resonances. The signal is extracted with a binned likelihood fit to the BDT output performed simultaneously in all channels (ee, e $\mu, \mu \mu)$ and in signal (1 jet 1 tag) and control regions ( 2 jets 1 tag, 2 jets 2 tags). Four variables are used to build the BDT output.

The main background is the $\mathrm{Z} / \gamma^{*}$ channel and data are used to correct the Missing transverse energy $\mathrm{E}_{T}^{\text {miss }}$ distribution not well modelled by MC in high pile-up periods. All the other backgrounds are negligible and extracted by simulation.

The distribution of the BDT output is shown in fig. 5 for data and $\mathrm{MC}$ in the signal region. Also if the variable is not very well picked around one for the tW channel, the signal is clearly visible over the background and can be extracted by the binned likelihood fit.

The extracted cross section is:

$$
\sigma=15_{-4}^{+5} p b
$$

corresponding to a measurement at $4 \sigma$ level.

Also in this case under the SM assumptions we can extract $\left|\mathrm{V}_{t b}\right|$ :

$$
\left|V_{t b}\right|=1.01_{-0.13}^{+0.16} \pm 0.04 \text { (theo.). }
$$

or

$$
\left|V_{t b}\right|>0.79 @ 95 \% C L \text { for }\left|V_{t b}\right| \text { in }[0,1]
$$

The main source of systematic error is the Jet Energy scale.

\section{4 single top t-channel charge ratio}

The different amount of $\mathrm{u}$ and d quark inside the proton generates an asymmetry in the production of $\mathrm{t}$ and $\bar{t}$ quarks. 


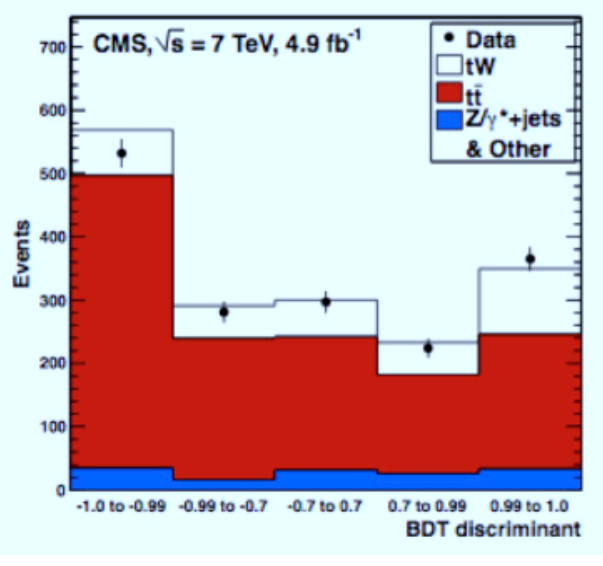

Figure 5. distribution of the BDT discriminant variable for data and $\mathrm{MC}$ in the signal region optimized for the $\mathrm{tW}$ analysis

The $\mathrm{t}$ and $\bar{t}$ charge can be deduced by the charge of the lepton emitted in the final state. The measurement of the asymmetry can constrain different PDF models and can also be sensitive to physics beyond standard model. According to [13] the expected value of the ratio in the Standard Model is

$$
R_{t-c h .}=\sigma_{t-c h ., t o p} / \sigma_{t-c h ., \text { anti-top }}=1.84
$$

The analysis [14] performed in CMS uses a data sample of $12.2 \mathrm{fb}^{-1}$ at $8 \mathrm{TeV}$ center of mass energy and is based on the $\left|\eta_{j^{\prime}}\right|$ analysis already described in section 2 . An extended likelihood function is defined including the yield and the probability distribution functions for signal and background and a fit to the $\left|\eta_{j^{\prime}}\right|$ distribution is performed simultaneously for muons and electrons and for charge plus and minus, in the signal region. The signal region is defined as the region with 2 jets (with $\mathrm{p}_{T}>30$ $\mathrm{GeV} / \mathrm{c}$ ) and 1 jet tagged as b-jet with the additional requirement that the invariant mass of the system composed by the lepton the neutrino and the b-jet is around the top mass $\left(130<\mathrm{m}_{l v b}<220 \mathrm{GeV} / \mathrm{c}^{2}\right)$.

The cross sections measured for the t-channel top and anti-top and for the ratio are:

$$
\begin{gathered}
\sigma_{t-\text { ch,top }}=49.9 \pm 1.9(\text { stat. }) \pm 8.9(\text { syst. }) p b \\
\sigma_{t-\text { ch,anti-top }}=28.3 \pm 2.4(\text { stat. }) \pm 4.9(\text { syst. }) p b \\
\left.\left.R_{t-\text { ch. }}=1.76 \pm 0.15 \text { (stat. }\right) \pm 0.22 \text { (syst. }\right)
\end{gathered}
$$

The results are compared to the standard model predictions with different pdf models in fig. 6 .

The extraction of the $\mathrm{W}+$ jets and $t \bar{t}$ backgrounds are the main source of systematic error together with the pdf uncertainty.

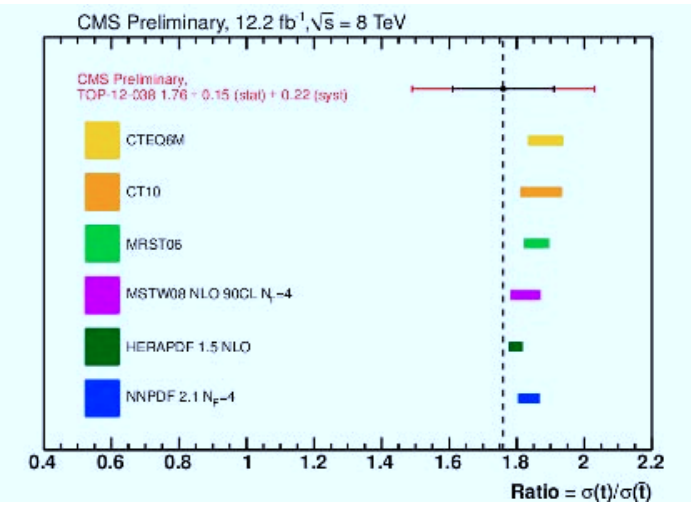

Figure 6. comparison between CMS experimental results and Standard model predictions with different PDF models of the single top t-channel charge ratio

\section{Conclusion}

CMS is exploring the large realm of single top physics. The t-channel cross section has been measured both at 7 and $8 \mathrm{TeV}$, while for the tW channel evidence at $4 \sigma$ level has been achieved at $7 \mathrm{TeV}$ center of mass energy. From these measurement has been possible to put limits on $\left|\mathrm{V}_{t b}\right|$. In particular from the t-channel cross section is possible to measure $\left|\mathrm{V}_{t b}\right|$ with a precisiom of $5 \%$. The charge ratio in the t-channel cross section has been measured at $8 \mathrm{TeV}$. CMS has also measured for the first time the W-helicity in single top topology. The reuslts have not been included in this paper but can be found in [15]. More data are under study and new results are expected in the near future.

\section{References}

[1] CMS Collaboration, "The CMS experiment at the CERN LHC", J.Instrum. 3, (2008) S08004,doi:10.1088/1748-0221/3/08/S08004.

[2] Kidonakis N., Phys. Rev. D 83 091503, (2010) (Preprint 1103.2792 [hep-ph])

[3] CMS Collaboration, "b-jet identification in the CMS experiment", CMS Physics Analysis Summary CMS-PAS-BTV-11-004 (2011), URL http://cdsweb.cern.ch/record/1427247

[4] CMS Collaboration, JHEP12 (2012) 35

[5] CMS Collaboration, "Measurement of the singletop-quark t-channel cross section in pp collisions at $\sqrt{s}=8$ TeV”, CMS Physics Analysis Summary CMS-PAS-TOP-12-011, (2012) URL https://cds.cern.ch/record/1478935

[6] E.T. Jaynes, "Probability theory: the logic of science", Cambridge University Press, Cambridge U.K. (2003).

[7] L. Lyons, D. Gibaut and P. Clifford, "How to combine correlated estimates of a single physical quantity”, Nucl. Instrum. Meth. A 270 (1988) 110

[8] S. Frixione et al., "Single-top hadroproduction in association with a W boson", JHEP 07 (2008) 029 
[9] T. M. P. Tait, "The t W-mode of single top production", Phys. Rev. D 61 (1999) 034001

[10] CMS Collaboration, Phys. Rev. Lett. 110 (2012) 022003

[11] Group T E W (CDF and D0 Collaborations), Preprint 0908.2171 [hep-ex] (2009)

[12] Campbell J. M., Huston J. W. and Stirling W. J., Rept. Prog. Phys. (2007) 70-89 (Preprint hep$\mathrm{ph} / 0611148$ )
[13] N. Kidonakis, "Differential and total cross sections for top pair and single top production", arXiv: 1205.3453

[14] CMS Collaboration, "Measurement of the single-top t-channel charge ratio at $\sqrt{s}=8 \mathrm{TeV}$ ", CMS Physics Analysis Summary CMS-PAS-TOP-12-038, (2012)

[15] CMS Collaboration, "W-helicity measurement in single top events topology", CMS Physics Analysis Summary CMS-PAS-TOP-12-020, (2012) 